\title{
Addressing Gaps in Interpreter Use: Time for Implementation Science Informed Multi-Level Interventions
}

\author{
Elaine C. Khoong, $M D, M S^{1,2}$ and Alicia Fernandez, $M D^{1,2,3}$ \\ 'Division of General Internal Medicine at Zuckerberg San Francisco General Hospital, Department of Medicine, University of California San \\ Francisco, San Francisco, CA, USA; ${ }^{2}$ UCSF Center for Vulnerable Populations at Zuckerberg San Francisco General Hospital, University of California \\ San Francisco, San Francisco, CA, USA: ${ }^{3}$ UCSF Latinx Center of Excellence, University of California San Francisco, San Francisco, CA, USA.
}

J Gen Intern Med 36(11):3532-6

DOI: $10.1007 / \mathrm{s} 11606-021-06823-4$

(c) Society of General Internal Medicine 2021

A 2017 report estimated nearly 258 million migrants globally. ${ }^{1}$ This global movement means that language barriers to healthcare have become more widespread. In the USA, nearly 25.5 million individuals speak English less than "very well." ${ }^{2}$ The prevalence of language barriers has important implications for all aspects of the Quadruple Aim for highquality healthcare, ${ }^{3}$ from the Triple Aim of healthcare costs, population health, and patient care experience to the additional aim of clinician wellness.

As US-based researchers, we focus on healthcare disparities among limited English proficient (LEP) patients in the USA, but similar disparities exist for all immigrants who do not speak the local dominant language. These health disparities include worse patient-reported and health outcomes as well as increased utilization. ${ }^{4,5}$ Ample research has shown that language access services, whether via language-concordant clinicians or professional interpreters, are crucial to ensure more equitable care and outcomes for immigrant patients. ${ }^{6}$ Though language-concordant clinicians can yield better outcomes than interpreted care, ${ }^{7}$ it is impossible to ensure languageconcordant care for the 350 languages within the USA, much less the 7000+ languages globally.

For decades, we have known that professional interpreters (versus family or ad hoc staff) improve patient-reported and clinical outcomes. ${ }^{6}$ Despite this evidence, professional interpreters are underutilized. ${ }^{8,9}$ Patients and clinicians report that interpreters are often not employed in clinical encounters, despite being free in many systems and mandated in the USA by statutory requirements for large healthcare systems. ${ }^{10}$ The development of newer tools, such as video interpretation, has lowered barriers to working with interpreters and enhanced

Received February 3, 2021

Accepted April 8, 2021

Published online May 4, 2021 the quality of interpretation; yet their adoption has proceeded unevenly across and within healthcare systems. Despite extensive research on disparities for patients with language barriers, conclusive evidence that interpreters help mitigate these gaps, and novel ways of reaching interpreters efficiently, progress has been limited on increasing interpreter use.

\section{THE IMPLEMENTATION GAP IN INTERPRETER USE}

One of the reasons for continued low rates of interpreter adoption is that most interventions have focused primarily on targeting individuals (such as clinicians or patients). Efforts focused on increasing clinician knowledge about interpreter use have largely been ineffective at sustaining increased interpreter use. ${ }^{11}$ Interventions to facilitate interpreter access for patients or clinicians by increasing device deployment have shown mixed results at best. ${ }^{12,13}$

Although these strategies make sense, similar to other evidence-based interventions, multiple barriers inhibit adoption of interpreters; therefore, multifaceted interventions are needed and more effective. ${ }^{11}$ Implementation science, which studies how best to implement evidence-based interventions, can inform analyses on why interpreter adoption is low (i.e., the implementation gap) and design of solutions to address this implementation gap. By using implementation science frameworks and tools, researchers and leaders can better comprehensively understand the factors that impact interpreter utilization.

In this paper, we apply the Consolidated Framework for Implementation Research (CFIR), ${ }^{14}$ a commonly used implementation science framework, to explain the interpreter implementation gap. This comprehensive framework is particularly useful for understanding low adoption rates of complex, multistep interventions such as interpreter implementation. CFIR includes five main constructs: intervention characteristics, individuals, inner setting, implementation process, and outer setting. (See Table 1 columns 1 and 2.) Within the context of this intervention, intervention characteristics are focused on features of interpreters. Key individuals are clinicians and patients. The inner setting is the healthcare organization while the outer setting includes external factors outside 
Table 1 Factors that impact interpreter implementation

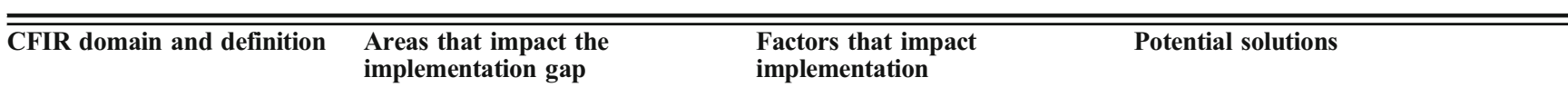

Intervention characteristics:

features of an intervention that

influence implementation

$\begin{array}{ll}\text { A. Interpreters } & \text {-Cost of interpreters } \\ & \text {-Variability in interpreter quality } \\ & \text {-Availability of bilingual } \\ & \text { clinicians }\end{array}$

Individual: characteristics of individuals involved in implementation that influence implementation

B. Clinicians and patients

Inner setting: features of the implementing organization that influences implementation

\section{Healthcare organizations}

D. Process for implementing interpreters

Implementation process: strategies or tactics that influence implementation
Outer setting: features of external context or environment that influence implementation
E. External influencers, such as healthcare payers, policy makers, digital health companies, and medical litigation
-Patient or clinician beliefs on value of professional interpreter use

-Self-efficacy about using of interpreters

-Interpreter access infrastructure, such as ease of access to phone, video, or in-person interpreters -Culture towards importance of language access

-Climate for adopting new

language access tools

- Planning process for implementation, availability, and access across all clinical settings -Whether clinicians, interpreters, patients, and other stakeholders are engaged in the implementation process

-Evaluating adoption or impact of language access implementation strategies

-Legal requirements and regulatory enforcement

-Financial incentives for interpreter use (or penalization of lack of use)

-Standard practice in leading healthcare organizations -Digital tools for interpreter access
-National standards for medical interpreter certification to ensure quality

-Training/support of interpreters in specific clinical areas (e.g., palliative care, COVID19) with higher need for nuanced communication

-Increase number of bilingual clinicians -Clinician training on effective use of interpreters for all members of the healthcare team and at all levels of training -Patient empowerment to request interpreter by providing easier access to interpreters, posting signage in multiple languages explaining patients' rights, and connecting patients to language-concordant navigators -Organizational policies that communicate expectations for interpreter use

-Organizational metrics for clinician evaluation of appropriate interpreter use -Organizational investment in mechanisms that ease access including equipment purchase and distribution, number of interpreters, and deployment of interpreters for in-person encounters

-Easing ability to monitor, measure, and evaluate adequate interpreter use through -Human-centered design of interpreter access workflow for clinicians and patients

-Regulatory policies governing interpreter use in all settings by all healthcare team members

-Auditing of adherence to established policies

-Development of digital tools and standards that expedite and ease interpreter access, request, and documentation

-Digital tools that automate auditing of adherence to policies

-Financial incentives for appropriate interpreter use healthcare organizations, such as healthcare payers or policy makers. The implementation process focuses on how interpreters are implemented.

To illustrate how all five constructs impact interpreter implementation, we draw on experiences from our institution, which cares for many patients with limited English proficiency, to create a journey map of a typical encounter with an LEP patient (Fig. 1). This journey map starts at recognizing an interpreter need to requesting the interpreter to the interpreted encounter to documenting the interpreter use. We then identify the CFIR domain(s) most relevant for each step of the journey. This complex, multi-step journey illustrates how all CFIR domains apply to interpreter implementation.

\section{KNOWN AND POSITED BARRIERS TO INTERPRETER USE}

The first factor that impacts interpreter use is the intervention itself, such as the cost and quality of interpreters (A in Fig. 1/
Table 1). Given the current reliance on people (as opposed to machines) for interpretation services, the cost of interpreters may be perceived as high, particularly if interpreter use expands to meet clinical need. There may also be quality concerns. If during the interaction, clinicians and patients perceive interpreter quality as subpar, in the future each party may be less likely to work with interpreters.

Clinicians' and patients' own beliefs (B in Fig. 1/Table 1) are also important factors. Patients may not feel empowered to request an interpreter when there are communication gaps or may prefer to use family members or their own limited language skills. ${ }^{15}$ This is particularly common when privacy concerns are paramount, such as in stigmatized clinical scenarios or when patient and interpreter are members of a small community. Clinicians may also feel that an interpreter would not add to a simple interaction and choose to get by using their own or the patient's language skills. ${ }^{10}$ Alternatively, if clinicians lack the skills and knowledge to work with interpreters 


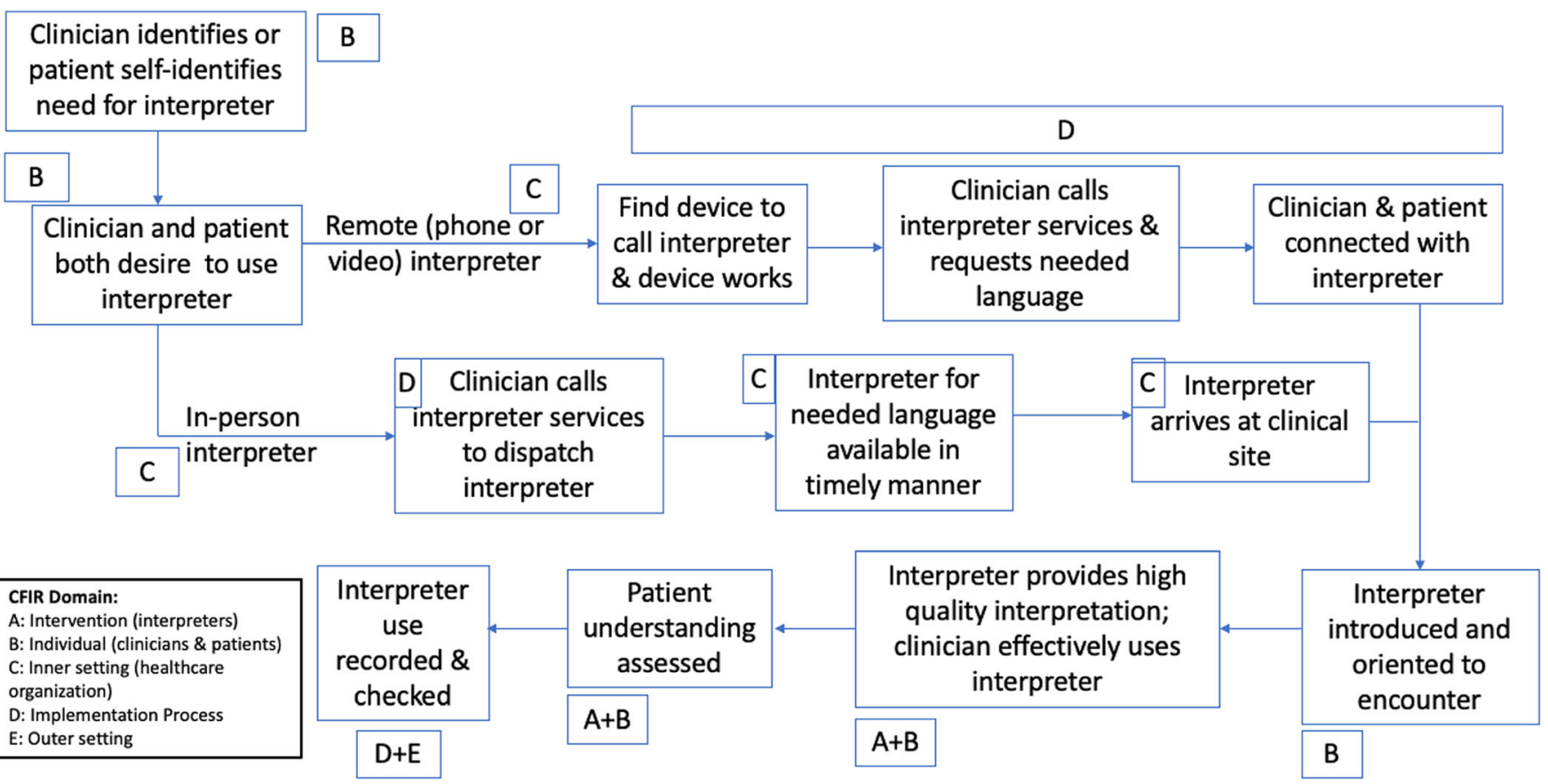

Fig. 1 Example journey map of pathway to using an interpreter for a limited English proficient patient. This figure demonstrates the steps that may be required to request and work with an interpreter as well as to document the interpreter presence during the encounter. Each of these steps aligns with one of the five implementation factors identified by the consolidated framework for implementation research (CFIR): (A) intervention (interpreters); (B) individuals (clinicians and patients); (C) inner setting (healthcare organization); (D) implementation process; (E) outer setting

effectively, they may underestimate the impact of professional interpreters on common clinical scenarios such as eliciting patient symptoms/values or reconciling medications, and hence choose not to work with interpreters. ${ }^{10,16}$

Organizational culture and policies (C in Fig. 1/Table 1) play a large role in interpreter use. Organizations may undervalue interpreter use relative to other priorities. This can lead to cost discussions about language access services that are removed from discussions about costs incurred in other clinical interactions. By underinvesting in infrastructure to make interpreters more easily accessible at the point of clinical care, organizations disincentivize clinicians from incorporating professional interpreters into encounter. Underinvestment in infrastructure to ease access, deployment, and scheduling of interpreters is common due to organizational culture that values immigrant patients less than other patients or that undervalues clinician time.

The process that organizations use to implement interpreters (D in Fig. 1/Table 1) is also impactful. Often decisions about interpreter access and availability are made without stakeholder input. As a result, clinicians and patients may experience delays or inconveniences when requesting interpreters. ${ }^{17}$ Seemingly small delays can be deal-breaking for busy clinicians as can "extra" effort, such as the need to manually enter medical record numbers. The ease of obtaining an interpreter is seldom monitored or assessed by organizations despite its importance in ensuring the success of language access efforts.

Finally, external organizations (E in Fig. 1/Table 1) impact interpreter uptake through a variety of mechanisms.
Regulatory agencies can enforce and audit healthcare organizations' use of interpreters. Healthcare payers could create financial incentives for using interpreters. Digital health companies could develop technological solutions to improve ease of accessing interpreters.

\section{INTERVENTIONS TO INCREASE INTERPRETER USE}

Given the breadth of factors that impact interpreter use when viewed from an implementation science lens, it is clear that multimodal efforts are necessary to improve language access. One example of an effective multimodal intervention is documentation of informed consent discussion for patients with language barriers. At our institution, this took the form of efforts to educate patients and clinicians, interventions to improve interpreter access, and importantly, also enforcement of interpreter use during informed consent discussions by regulatory agencies. ${ }^{18}$ As the absence of documented language access services can be the basis of a legal challenge, hospital risk management services have also weighed in on the importance of necessary changes. As a result of this multi-level, multiple-stakeholder approach, there have been significant gains at improving interpreter use during informed consent discussions. This successful effort illustrates that language access advocates must pursue several solutions concurrently (Table 1).

Interpreter cost and variation in quality (CFIR domain: intervention characteristic) impact interpreter use. In the USA, advocates for LEP patients should push for the enforcement of 
national standards for medical interpreter certification. Efforts to increase certification and deployment of bilingual staff and clinicians are also helpful, and depending on the wage structure, may address cost concerns related to interpreter use. Recruitment and training of bilingual clinicians may improve the accessibility of language-concordant care and quality of care as well as reduce the need for as many interpreters.

Robust efforts to address clinician and patient knowledge and behaviors (CFIR domain: individuals) should continue and be pursued via approaches most likely to result in behavior change. For example, rather than hanging posters that inform patients of their rights to language access, new patients can be assigned to language-concordant health navigators that inform patients of their rights and make it easy for patients to indicate the need for interpreters. Interpreters can be automatically scheduled for patients with a non-English (or non-dominant language) preference. Students and practicing clinicians are motivated by participating in simulated encounters with patients with language barriers, as opposed to a single, readily forgettable lecture. ${ }^{19}$

Healthcare organizations (CFIR domain: inner setting) have the potential to significantly impact interpreter use by establishing written policies that prioritize interpreter use. For example, organizations can communicate an expectation that every hospitalized patient with language barriers will engage in at least one interpreted conversation with their treating clinician each day. Since organizations will need to audit their clinicians to ensure adherence to institutional policies, fields to document interpreter use should be standard in electronic medical records. Healthcare systems should also create a process for formal assessment of language proficiency among bilingual staff and clinicians. This should also be accompanied by strategies to proactively hire, support, and compensate staff and clinicians that have the language skills to provide language-concordant care. ${ }^{20}$ Moreover, organizations should devote resources to increasing interpreter infrastructure (e.g., number of interpreters, number of interpreter devices, ease of accessing interpreter services).

The process for accessing interpreters and documenting their use (CFIR domain: process) is ripe for innovation and change. In many healthcare systems, this process is rarely designed with the end user in mind. For example, in our own institutions, we frequently have to use our own devices to call an interpreter or acquire a device from another location since there is not always a device available bedside. It is also not uncommon for clinicians to experience delays as they request the desired language and wait for an interpreter that speaks that language. Furthermore, it is difficult for many health systems to audit if interpreters were actually used, which hinders quality improvement efforts. In many organizations, the process for acquiring an interpreter needs to be revisited and redesigned for ease of use. Health information companies and human-centered design processes can play a crucial role in this effort.
External influences (CFIR domain: outer setting) will continue to be crucial, if not the most crucial factor, as demonstrated by the success at increasing interpreter use during informed consent conversations. Although regulatory requirements exist in the USA surrounding interpreter use, unless regulations are enforced, the only impetus for change comes from lawsuits. Regulatory bodies, such as the Joint Commission, should audit organizations on their use of interpreters. Healthcare payers should also provide financial incentives/ support for provision of language access. ${ }^{21}$ As healthcare delivery increasingly occurs through telemedicine, industry stakeholders need to develop technical solutions that ease interpreter access and use. Data standards organizations should establish standards for how interpreter requests are communicated from a healthcare provider to an interpreting agency and how remote interpreters are included in telemedicine encounters. Right now, these requests are rarely automated and require healthcare providers and interpreters to manually create a process that works for their specific relationship.

Language barriers in healthcare are inevitable in a globalized world, but its associated unequal care and outcomes are not inevitable. Addressing these disparities necessitates a multi-level approach informed by implementation science theory, such as the CFIR-based approach we use here. Guided by our experience, we outlined several strategies that should be concurrently pursued. While strategies will need to be adapted to local settings, an implementation science approach is necessary to facilitate meaningful progress on reducing these disparities. We must act now on these multi-level strategies; otherwise, language barriers and inequities in healthcare will only increase as global migrations grow.

Corresponding Author: Elaine C. Khoong, MD, MS; Division of General Internal Medicine at Zuckerberg San Francisco General Hospital, Department of Medicine, University of California San Francisco, San Francisco, CA, USA (e-mail: elaine.khoong@ucsf.edu).

Funding Dr. Khoong is supported by the National Heart Lung and Blood Institute of the NIH under Award Number K12HL138046. Dr Fernandez is supported by NIH K24DK102057 and HRSA D34HP31878.

\section{Declarations:}

Conflict of Interest: None.

Disclaimer.

The content is solely the responsibility of the authors and do not necessarily represent the official views of the NIH.

\section{REFERENCES}

1. United Nations. Migration. https://www.un.org/en/sections/issuesdepth/migration/index.html. Published 2018. Accessed June 17, 2019.

2. United States Census Bureau. Language Use. https://www.census.gov/ topics/population/language-use/about/faqs.html. Published 2017. Accessed September 23, 2018 
3. Bodenheimer T, Sinsky C. From Triple to Quadruple Aim: Care of the Patient Requires Care of the Provider. Ann Fam Med. 2014;12(6):573576. doi:https://doi.org/10.1370/afm.1713

4. Parker MM, Fernández A, Moffet HH, Grant RW, Torreblanca A Karter AJ. Association of Patient-Physician Language Concordance and Glycemic Control for Limited-English Proficiency Latinos With Type 2 Diabetes. JAMA Intern Med. 2017;177(3):380. doi:https://doi.org/10. $1001 /$ jamainternmed.2016.8648

5. Ngai KM, Grudzen CR, Lee R, Tong VY, Richardson LD, Fernandez A The Association Between Limited English Proficiency and Unplanned Emergency Department Revisit Within 72 Hours. Ann Emerg Med. 2016;68(2):213-221. doi:https://doi.org/10.1016/j.annemergmed.2016. 02.042

6. Karliner LS, Jacobs EA, Chen AH, Mutha S. Do professional interpreters improve clinical care for patients with limited english proficiency? A systematic review of the literature. Health Serv Res. 2007;42(2):727754. doi:https://doi.org/10.1111/j.1475-6773.2006.00629.x

7. Ngo-Metzger Q, Sorkin DH, Phillips RS, et al. Providing High-Quality Care for Limited English Proficient Patients: The Importance of Language Concordance and Interpreter Use. J Gen Intern Med. 2007;22(S2):324330. doi:https://doi.org/10.1007/s11606-007-0340-Z

8. Schulson LB, Anderson TS. National Estimates of Professional Interpreter Use in the Ambulatory Setting. J Gen Intern Med. November 2020:1-3. doi:https://doi.org/10.1007/s11606-020-06336-6

9. Diamond L, Wilson-Stronks A, Jacobs E. Do Hospitals Measure up to the National Culturally and Linguistically Appropriate Services Standards? Med Care. 2010. doi:https://doi.org/10.1097/MLR. Ob013e3181f380bc

10. Diamond LC, Schenker Y, Curry L, Bradley EH, Fernandez A. Getting By: Underuse of interpreters by resident physicians. J Gen Intern Med 2009;24(2):256-262. doi:https://doi.org/10.1007/s11606-008-0875-7

11. Taira BR, Kim K, Mody N. Hospital and Health System-Level Interventions to Improve Care for Limited English Proficiency Patients: A Systematic Review. Jt Comm J Qual patient Saf. 2019;0(0). doi:https:// doi.org/10.1016/j.jcjq.2019.02.005

12. Lee JSJS, Nápoles A, Mutha S, et al. Hospital discharge preparedness for patients with limited English proficiency: A mixed methods study of bedside interpreter-phones. Patient Educ Couns. 2018;101(1):25-32. doi:https://doi.org/10.1016/j.pec.2017.07.026

13. Tuot DS, Lopez M, Miller C, Karliner LS. Impact of an Easy-Access Telephonic Interpreter Program in the Acute Care Setting: An Evaluation of a Quality Improvement Intervention. Jt Comm $J$ Qual Patient Saf. 2012;38(2):81-AP21. doi:https://doi.org/10.1016/S1553-7250(12) 38011-2

14. Damschroder LJ, Aron DC, Keith RE, Kirsh SR, Alexander J a, Lowery JC. Fostering implementation of health services research findings into practice: a consolidated framework for advancing implementation science. Implement Sci. 2009;4(1):50. doi:https://doi.org/10.1186/17485908-4-50

15. Ragavan MI, Cowden JD. The Complexities of Assessing Language and Interpreter Preferences in Pediatrics. Heal Equity. 2018;2(1):70-73. doi:https://doi.org/10.1089/heq.2017.0057

16. Karliner LS, Pérez-Stable EJ, Gildengorin G. The Language Divide The Importance of Training in the Use. J GEN INTERN MED. 2004;19:175183. www.jgim.org. .

17. Burkle CM, Anderson KA, Xiong Y, Guerra AE, Tschida-Reuter DA. Assessment of the efficiency of language interpreter services in a busy surgical and procedural practice. BMC Health Serv Res. 2017;17(1):456. doi:https://doi.org/10.1186/s12913-017-2425-7

18. Lee JS, Pérez-Stable EJ, Gregorich SE, et al. Increased Access to Professional Interpreters in the Hospital Improves Informed Consent for Patients with Limited English Proficiency. $J$ Gen Intern Med. 2017;32(8):863-870. doi:https://doi.org/10.1007/s11606-017-3983-4

19. Diamond LC, Jacobs EA. Let's Not Contribute to Disparities: The Best Methods for Teaching Clinicians How to Overcome Language Barriers to Health Care. J Gen Intern Med. 2010;25(S2):189-193. doi:https://doi. org/10.1007/s11606-009-1201-8

20. Fernández A, Pérez-Stable EJ. ¿Doctor, habla español? Increasing the Supply and Quality of Language-Concordant Physicians for SpanishSpeaking Patients. J Gen Intern Med. 2015;30(10):1394-1396. doi:https://doi.org/10.1007/s11606-015-3436-x

21. Shah SA, Velasquez DE, Song Z . Reconsidering Reimbursement for Medical Interpreters in the Era of COVID-19. JAMA Heal Forum. 2020;1(10):e201240. doi:https://doi.org/10.1001/jamahealthforum. 2020.1240

Publisher's Note: Springer Nature remains neutral with regard to jurisdictional claims in published maps and institutional affiliations. 